\title{
ARTICLE
}

\section{Sarsasapogenin improves adipose tissue inflammation and ameliorates insulin resistance in high-fat diet-fed C57BL/6 J mice}

\author{
Yan-yan $\mathrm{Yu}^{1,2}$, Shi-chao Cui ${ }^{1}$, Tian-nan Zheng ${ }^{1,2}$, Hai-jian Ma ${ }^{1}$, Zhi-fu Xie ${ }^{1}$, Hao-wen Jiang ${ }^{1}$, Yu-feng $\mathrm{Li}^{1,2}, \mathrm{Ke}^{-x i n} \mathrm{Zhu}^{1,2}$, \\ Cheng-gang Huang ${ }^{1}$, Jia $\mathrm{Li}^{1}$ and Jing-ya $\mathrm{Li}^{1}$
}

\begin{abstract}
Insulin resistance is a major cause of type 2 diabetes and metabolic syndrome. Macrophage infiltration into obese adipose tissue promotes inflammatory responses that contribute to the pathogenesis of insulin resistance. Suppression of adipose tissue inflammatory responses is postulated to increase insulin sensitivity in obese patients and animals. Sarsasapogenin (ZGY) is one of the metabolites of timosaponin Alll in the gut, which has been shown to exert anti-inflammatory action. In this study, we investigated the effects of ZGY treatment on obesity-induced insulin resistance in mice. We showed that pretreatment with ZGY (80 $\mathrm{mg} \cdot \mathrm{kg}^{-1} \cdot \mathrm{d}^{-1}$, ig, for 18 days) significantly inhibited acute adipose tissue inflammatory responses in LPS-treated mice. In high-fat diet (HFD)-fed obese mice, oral administration of ZGY $\left(80 \mathrm{mg} \cdot \mathrm{kg}^{-1} \cdot \mathrm{d}^{-1}\right.$, for 6 weeks) ameliorated insulin resistance and alleviated inflammation in adipose tissues by reducing the infiltration of macrophages. Furthermore, we demonstrated that ZGY not only directly inhibited inflammatory responses in macrophages and adipocytes, but also interrupts the crosstalk between macrophages and adipocytes in vitro, improving adipocyte insulin resistance. The insulin-sensitizing and anti-inflammatory effects of ZGY may result from inactivation of the IKK /NF-KB and JNK inflammatory signaling pathways in adipocytes. Collectively, our findings suggest that ZGY ameliorates insulin resistance and alleviates the adipose inflammatory state in HFD mice, suggesting that ZGY may be a potential agent for the treatment of insulin resistance and obesity-related metabolic diseases.
\end{abstract}

Keywords: insulin resistance; sarsasapogenin; adipose tissue inflammation; macrophage-adipocyte crosstalk; IKK/NF-KB; JNK

Acta Pharmacologica Sinica (2021) 42:272-281; https://doi.org/10.1038/s41401-020-0427-1

\section{INTRODUCTION}

Insulin resistance is the earliest detectable metabolic disorder in individuals who eventually develop type 2 diabetes mellitus (T2D) [1]. Insulin resistance is also a critical risk factor for nonalcoholic fatty liver disease (NAFLD), atherosclerosis (AS), and cardiovascular disease (CVD) [2]. The mechanisms underlying insulin resistance are multifactorial, and the most dominant reason for insulin resistance is obesity [3-5]. Inflammation links obesity and insulin resistance. Obese animals and patients have increased circulating inflammatory markers such as tumor necrosis factor (TNF) a, interleukin (IL) 6, IL-1 $\beta$, and monocyte chemoattractant protein (MCP) 1, and reduced plasma levels of anti-inflammatory cytokines such as adiponectin and IL-10 [6-8]. The initiation of chronic inflammation is characterized by massive infiltration of macrophages in obese adipose tissues [3, 9, 10]. Adipose tissue and adipose tissue macrophages are the main sources of proinflammatory molecules [9]. Endocrine-mediated crosstalk between adipose tissue, the liver, and skeletal muscle leads to local and systemic inflammation, and insulin resistance through autocrine and paracrine signaling [1, 10-12]. In addition, infiltrated and innate macrophage phenotypic switching from an antiinflammatory $\mathrm{M} 2$ phenotype to a proinflammatory $\mathrm{M} 1$ polarization phenotype [13], and the interaction between adipocytes and integrated macrophages in progressive obesity exacerbate adipose tissue inflammatory responses and contribute to insulin resistance $[8,9]$. Activation of the inhibitor of nuclear factor-KB (IKK)/nuclear factor-KB (NF-KB) and c-Jun $\mathrm{N}$ terminal kinase (JNK) pathways also lead to insulin resistance by promoting inflammatory responses and blocking the insulin signaling pathway [3, 12]. Clinical and animal model studies have used either small molecule antiinflammatory approaches or biological agents that target inflammation to attenuate insulin resistance and T2D $[4,14]$.

Timosaponin Alll is one of the main constituents of the common herbal medicine Anemarrhena asphodeloides [15], and sarsasapogenin (ZGY) is one of the metabolites of timosaponin Alll in the gut [16]. ZGY improves learning and memory, has antitumor effects and inhibits inflammatory responses [17-19]. A recent study found that oral administration of timosaponin Alll and its gut metabolite ZGY suppresses 2,3,4-trinitrobenzene sulfonic acid (TNBS)-induced colitis in mice by inhibiting NF-KB and mitogen activated protein kinase (MAPK) activation. Notably, the anti-inflammatory effects of ZGY are more potent than those of timosaponin Alll [16]. However, the effects of ZGY on obesity-induced adipose tissue inflammation and insulin resistance have not been investigated. Our study

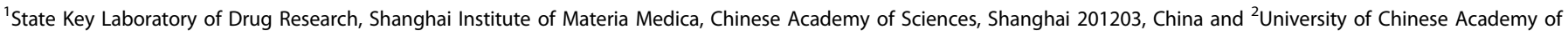
Sciences, Beijing 100049, China

Correspondence: Jia Li (jli@simm.ac.cn) or Jing-ya Li (jyli@simm.ac.cn)
}

Received: 11 February 2020 Accepted: 20 April 2020

Published online: 22 July 2020 
demonstrated for the first time that oral administration of ZGY improved insulin resistance in high-fat diet-induced obese mice and alleviated adipose tissue inflammation. In vitro, ZGY exerted direct effects on macrophages and adipocytes and disturbed crosstalk between adipocytes and macrophages. These findings suggest that ZGY is a potential anti-inflammatory agent to prevent obesity-associated inflammatory metabolic diseases.

\section{MATERIALS AND METHODS}

Chemicals and reagents

Antibodies against F4/80 (\#70076), IKB kinase $\beta$ (IKK, \#8943), phospho-IKK a/ $\beta$ (Ser176/180) (p-IKK, \#2697), B-cell inhibitor alpha (IKB-a, \#4814), nuclear factor (NF-KB) (p65, \#4764), phospho-NF-KB p65 (Ser536) (NF-KB, \#3033), the stress-activated protein kinase/ Jun-aminoterminal kinase SAPK/JNK (JNK, \#9252), phosphor-SAPK/ JNK (Thr183/Tyr185) (p-JNK, \#9251), insulin receptor substrate 1 (IRS1, \#2382), Akt (\#4691), phospho-Akt (Ser473) (\#4060), phosphoAkt (Thr308) (\#13038), phospho-insulin receptor (IR) (Tyr1162) (\#3918), and glyceraldehyde-3-phosphate dehydrogenase (GAPDH, \#5174) were purchased from Cell Signaling Technology; IR (sc-711) was purchased from Abcam. Recombinant human insulin (Eli Lily) was purchased from Changzheng Hospital (Shanghai). ELISA kits that were used to measure of plasma parameters were as follows: insulin (Crystal (Chem, \#90080), resistin (BioTNT, \#EMR1001-1), leptin (BioTNT, \#90030), adiponectin (BioTNT, \#80569), mouse MCP1 (BioTNT, MEC1005), mouse IL-6 (BioTNT, MEC1008), mouse IL-1 $\beta$ (BioTNT, MEC1010), mouse TNF-a (BioTNT, MEC1003), and V-PLEX custom mouse cytokine (MSD, $\mathrm{K} 152 \mathrm{AOH}-1)$. Tumor necrosis factor (TNF) a and lipopolysaccharide (LPS) were obtained from SigmaAldrich. High-glucose Dulbecco's modified Eagle's medium (HGDMEM) was purchased from HyClone.

Sarsasapogenin (ZGY) was derived from timosaponin Alll from the common herbal medicine Anemarrhena asphodeloides, which was provided by Professor Cheng-gang Huang from Shanghai Institute of Materia Medica (Fig. 1).

\section{Animal model}

All animal experiments were approved by the Animal Care and Use Committee of the Shanghai Institute of Materia Medica, where the experiments were conducted. All animals were housed in a temperature-controlled room $\left(22 \pm 2^{\circ} \mathrm{C}\right)$ with a light/dark cycle of $12 \mathrm{~h}$. Male C57BL/6J mice were fed either a high-fat diet (HFD, 60\% calories from fat, $20 \%$ calories from protein, and $20 \%$ calories from carbohydrate; Research Diets) or a normal chow diet beginning at 6 weeks of age. After 10 weeks, the mice were randomized into three groups according to body weight and fasting plasma glucose. The vehicle group (HFD-Veh), ZGY group (HFD-ZGY, $80 \mathrm{mg} / \mathrm{kg}$ ), and metformin group (HFD-Met, $250 \mathrm{mg} / \mathrm{kg}$ ) were gavaged for 6 weeks, and the normal chow (NC) group was used as a control.

Acute inflammatory responses in mice induced by LPS injection Male mice at the age of 10 weeks were divided into four groups $(n=10)$ : the control group (Veh), LPS-treated group $(30 \mathrm{mg} / \mathrm{kg}$,

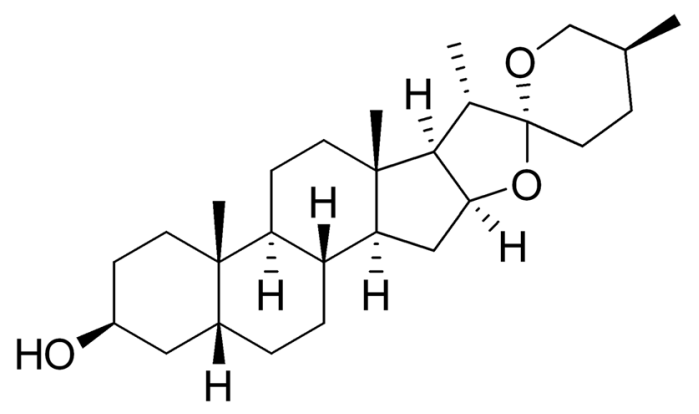

Fig. 1 Structure of sarsasapogenin.
LPS), and ZGY-treated groups ( $40 \mathrm{mg} / \mathrm{kg}$ and $80 \mathrm{mg} / \mathrm{kg} ;$ LPS-ZGY (40) and LPS-ZGY (80), respectively). Before LPS treatment, the mice were consecutively administered different concentrations of ZGY via oral gavage for 18 days. The Veh and LPS groups were administered the same volume of CMC-Na. After 18 days, the LPS, LPS-ZGY (40), and LPS-ZGY (80) group mice were treated by intraperitoneal injection with $30 \mathrm{mg} / \mathrm{kg}$ body weight LPS. The Veh group was administered the same volume of saline. After $6 \mathrm{~h}$ of LPS stimulation, all experimental mice were sacrificed; fasting blood was collected and centrifuged at $12,000 \times g$ for 3 min at $4{ }^{\circ} \mathrm{C}$ for subsequent analysis, and the whole liver and epididymal white adipose tissue (eWAT) were carefully harvested, immediately frozen in liquid $\mathrm{N}_{2}$, and stored at $-80^{\circ} \mathrm{C}$ for subsequent analysis.

Glucose tolerance test (GTT) and insulin tolerance test (ITT) After fasting for $6 \mathrm{~h}$, the mice were gavaged with glucose $(2 \mathrm{~g} / \mathrm{kg})$; glucose concentrations were measured before administration and $15,30,60,90$, and $120 \mathrm{~min}$ after glucose treatment. After fasting for $4 \mathrm{~h}$, the mice were i.p. injected with insulin $(0.75 \mathrm{U} / \mathrm{kg})$, and glucose concentrations were measured before administration and $15,30,60,90$, and $120 \mathrm{~min}$ after insulin injection.

\section{F4/80 immunohistochemistry}

The epididymal adipose tissue from the animals was dissected, fixed in $4 \%$ paraformaldehyde for $24 \mathrm{~h}$, embedded in paraffin, and sectioned for immunohistochemistry. The F4/80 antibody was used at a 1:200 dilution. Finally, the slides were scanned by a Pannoramic MIDI (3D HISTECH, Hungary). The images were evaluated and quantified with image analysis software (Quant Center, Densito Quant).

\section{ELISA measurement of adipokines}

Serum concentrations of TNF- $\alpha$, IL-1 $\beta$, IL-6, MCP-1, and IL-10 were detected with a V-PLEX custom mouse cytokine kit according to the manufacturer's instructions. The concentrations of each adipokine in the coculture medium of 3T3-L1 adipocytes and supernatant that was collected from RAW 264.7 cells were assayed using mouse ELISA kits, according to the manufacturer's protocol. Each concentration was determined using a standard curve.

RNA isolation and quantitative RT-PCR

Total RNA was isolated from cells and tissues using TRIzol reagent (Invitrogen). The cDNA was prepared using PrimeScript reverse transcriptase (TaKaRa) according to the manufacturer's instructions. After a 10-fold dilution, the cDNAs were amplified using $2 \times$ SYBR Green qPCR master mix (Biotool) and a Stratagene Mx3005P (Agilent Technologies). Primer sequence details are shown in the Supplementary Information.

Protein isolation and Western blotting

Proteins were extracted from cells and tissues with radioimmunoprecipitation assay (RIPA) buffer, denatured with loading buffer, resolved with $10 \%$ SDS-PAGE gels, electroblotted onto NC membranes, and then blocked with 5\% nonfat milk. The membranes were incubated with different primary antibodies, followed by incubation with the relative secondary antibodies, and the blots were analyzed by ImageJ.

3T3-L1 preadipocyte cell culture, differentiation, and treatment Mouse 3T3-L1 preadipocytes were purchased from Cell Bank (Chinese Academy of Sciences, Shanghai, China). The cells were cultured and differentiated into adipocytes as previously described. Briefly, fibroblasts were grown at $37^{\circ} \mathrm{C}$ in a $5 \% \mathrm{CO}_{2}$ atmosphere in HG-DMEM containing 10\% FCS and 1\% penicillin/ streptomycin (Invitrogen) (growth medium). 3T3-L1 cells were incubated for 2 days until reaching confluence. At this point (day $0)$, the cells were exposed to standard differentiation inducers (HG-DMEM containing $1 \mathrm{ng} / \mathrm{mL}$ DEX, $500 \mu \mathrm{M}$ IBMX, $278 \mu \mathrm{M}$ insulin, 
$0.1 \mu \mathrm{g} / \mathrm{mL}$ biotin and $10 \%$ FBS) for 3 days. The medium was then replaced with DMEM supplemented with $278 \mu \mathrm{M}$ insulin and $10 \%$ FBS and was changed every other day for the following 5 days. We used TNF-a to induce adipocyte inflammatory responses and insulin resistance. The mature 3T3-L1 cells were treated with TNF- $a$ alone $(100 \mathrm{ng} / \mathrm{mL})$ or TNF- $a$ with various concentrations of ZGY for $24 \mathrm{~h}$, and the cells were used for RNA extraction. The mature 3T3-L1 cells were pretreated with ZGY for $24 \mathrm{~h}$ before TNF-a $(100 \mathrm{ng} / \mathrm{mL})$ treatment for $30 \mathrm{~min}$, and cell lysates were obtained for inflammatory signaling pathway analyses. The mature 3T3-L1 cells were treated with TNF- $a$ alone $(100 \mathrm{ng} / \mathrm{mL})$ or TNF- $a$ with various concentrations of ZGY for $24 \mathrm{~h}$ to induce insulin resistance. Then, $100 \mathrm{nM}$ insulin was added to the medium during the final $30 \mathrm{~min}$, and cell lysates were obtained for insulin signaling pathway analyses.

Isolation and differentiation of inguinal white adipose tissue stromal vascular fraction (iWAT-SVF) cells

iWAT-SVF cells were isolated from 5-week-old male C57BL/6J mice as previously described [20]. In brief, inguinal adipose tissue was minced and digested with $10 \mathrm{mg} / \mathrm{mL}$ collagenase $\mathrm{D}$ (Roche) and $2.4 \mathrm{mg} / \mathrm{ml}$ dispase II (Roche) in PBS supplemented with 1\% BSA for $45 \mathrm{~min}$ at $37^{\circ} \mathrm{C}$, followed by quenching with complete medium. The digested tissue suspensions were centrifuged, washed and successively filtered through $100-$ and $40-\mu \mathrm{m}$ strainers (BD Biosciences), and then, the cells were plated onto $10-\mathrm{cm}$ dishes. iWAT-SVF cells were cultured in DMEM/F12 supplemented with $10 \%$ FBS (Gibco) and 1\% penicillin/streptomycin (Invitrogen). iWAT-SVF cells were plated onto 24-well plates until reaching confluence. Once the cells reached confluence, adipocyte differentiation and treatment with ZGY were performed in the same manner as that of 3T3-L1 cells.

Treatment of 3T3-L1 adipocytes with conditioned medium (CM) from LPS-activated RAW 264.7 macrophages

RAW 264.7 cells $\left(3 \times 10^{5}\right.$ cells/well in a 24-well plate) were incubated in $1 \mathrm{~mL} /$ well culture medium containing LPS $(100 \mathrm{ng} / \mathrm{mL})$ alone or LPS with ZGY for $24 \mathrm{~h}$. The CM was collected and transferred onto day $83 T 3-\mathrm{L} 1$ adipocytes $(0.5 \mathrm{~mL}$ of $\mathrm{CM} /$ well in a 24-well plate without any fresh medium). The mature 3T3-L1 cells were treated with macrophage-conditioned medium and were named the CM group. For the control condition, culture medium containing the same concentrations of LPS and ZGY but without any secreted factors from the macrophages was added to 3T3-L1 adipocytes on day 8 , and we named these treated $3 \mathrm{~T} 3-\mathrm{L} 1$ cells the A group. After $24 \mathrm{~h}$, the medium and cells were used for ELISA, RNA extraction, and Western blotting. For insulin signaling analysis, $100 \mathrm{nM}$ insulin was added to the medium during the final $30 \mathrm{~min}$.

Statistical analysis

Each experiment was performed independently three times. The results are presented as the means $\pm S E M$. Student's $t$-test (twosided) was used to test for significant differences between groups. $P<0.05$ was regarded as statistically significant.

\section{RESULTS}

ZGY inhibits acute adipose inflammatory responses induced by intraperitoneal injection of LPS in C57BL/6J mice

Some studies show that ZGY has anti-inflammatory activity [16]. We indicated that ZGY preferentially accumulated in the adipose tissue after a single oral administration (S Fig. 1), suggesting that adipose tissue may be the target of oral ZGY treatment. LPS is a bacterial endotoxin that is derived from the gut microbiota and is widely used to investigate the acute inflammatory response to infection in rodents [21, 22]. In our study, we found that LPS injection stimulated the release of cytokines, including TNF- $\alpha$, IL- $1 \beta$, and IL- 6 , and pretreatment of
C57BL/6J mice with ZGY at $80 \mathrm{mg} / \mathrm{kg}$ for 18 days improved the release of plasma TNF- $\alpha$, IL- $1 \beta$, and IL- 6 in plasma upon LPS treatment and increased the concentration of the antiinflammatory cytokine IL-10 (Fig. 2a). Adiponectin is an adipokine that is mainly secreted by adipose tissue and is a well-defined obesity marker that exerts multiple beneficial effects against inflammation and insulin resistance [23-25]. We found that LPS treatment decreased the plasma adiponectin level, and ZGY pretreatment showed slight protection against LPS-induced changes in adiponectin (Fig. 2a).

Adipose tissue is a secretory organ that secretes numerous cytokines that are involved in acute inflammatory responses. LPS binds to Toll-like receptors, initiates the IKK/NF-KB and JNK signaling pathways and promotes proinflammatory gene expression and secretion [26]. Our results showed that ZGY treatment suppressed LPS-induced expression of the proinflammatory genes TNF- $a, I L-1 \beta, I L-6, M C P-1$, Nos2, and COX2 in eWAT (Fig. 2b). We also observed a concomitant increase in the transcription of $\mathrm{M} 2$ markers, such as Arg1, Ym1, Fizz1, and IL-10 (Fig. 2C), and the LPSinduced reduction of adiponectin in eWAT was ameliorated (Fig. 2d). Moreover, ZGY decreased LPS-induced IKK, NF-KB, and JNK phosphorylation and increased the protein level of IKB- $\alpha$ in eWAT (Fig. 2e, f), suggesting that LPS-induced degradation of IKBa was abrogated. These results demonstrate that oral administration of ZGY displayed inhibitory effects on LPS-induced acute inflammatory responses, improved the inflammatory status of adipose tissue, and inactivated the IKK/NF-KB and JNK inflammatory signaling pathways.

Chronic ZGY treatment ameliorates insulin resistance in high-fat diet-induced mice

Next, we established an insulin resistance model by feeding C57BL/6J mice with a high-fat diet for 10 weeks, as evidenced by impaired glucose tolerance. The mice received daily gavage of 80 $\mathrm{mg} / \mathrm{kg}$ ZGY for 6 weeks to evaluate its protective effects against HFD-induced insulin resistance, and metformin (HFD-Met, $250 \mathrm{mg} /$ $\mathrm{kg}$ ) was used as a positive control. During the treatment, ZGY did not affect body weight gain or food intake (S Fig. 2a, b). At the end of the experiment, ZGY treatment did not change the weight of the liver, epididymal adipose tissue (eWAT), inguinal adipose tissue (iWAT), perirenal white adipose tissue (pWAT), kidney, or pancreas (S Fig. 2c). HFD caused a significant increase in plasma fasting insulin, and the administration of ZGY obviously restored hyperinsulinemia (Fig. 3a). The insulin tolerance test showed that ZGY treatment induced advanced insulin sensitivity, as evidenced by a lower area under the curve $\left(\mathrm{AUC}_{0-120}\right)$ than that of the HFDVeh group, and the HFD-Met group had significantly improved insulin sensitivity (Fig. 3b, c). These data suggest that ZGY has protective effects on insulin resistance in HFD mice. In addition, administration of ZGY improved the oral glucose tolerance of HFD mice, as evidenced by the decrease in the $A U C_{0-120}$ and the fasting blood glucose compared to those of the HFD-Veh group (Fig. 3d-f).

To identify the target peripheral organ of insulin sensitivity, the mice were intraperitoneally injected with insulin $(2 \mathrm{U} / \mathrm{kg})$, and the liver, skeletal muscle, and eWAT were harvested at $15 \mathrm{~min}$ for biochemical analyses of endogenous activity of the insulin signaling pathways. Insulin receptor (IR) tyrosine phosphorylation, which is one of the earliest responses to insulin binding, suggests an increase in insulin responsiveness. Phosphorylation of the serine/threonine kinase Akt at serine-473 (Ser ${ }^{473} \mathrm{Akt}$ ) and threonine-308 (Thr ${ }^{308} \mathrm{Akt}$ ), which is a subsequent step in this cascade, correlates with IR activation in tissue [12]. We found that the blunted insulin-stimulated phosphorylation of IR and Akt in eWAT (Fig. 3g, h), the liver (Fig. 3i, j), and muscle (Fig. 3k, I) of HFD mice was increased after ZGY treatment, providing a biochemical correlation for increased in vivo insulin sensitivity. 
a
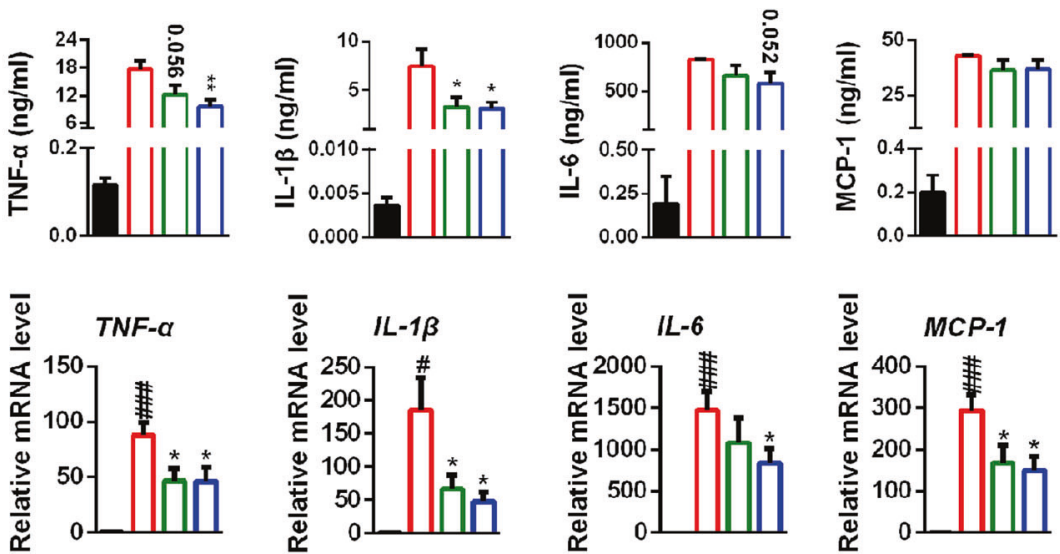

C

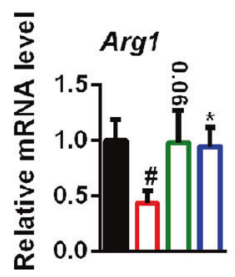

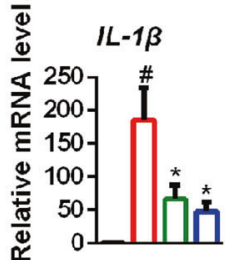

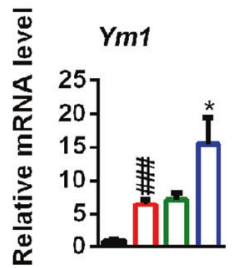

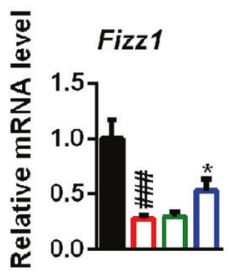

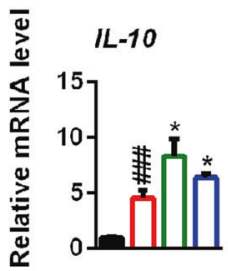

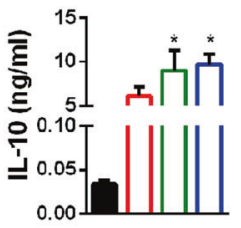
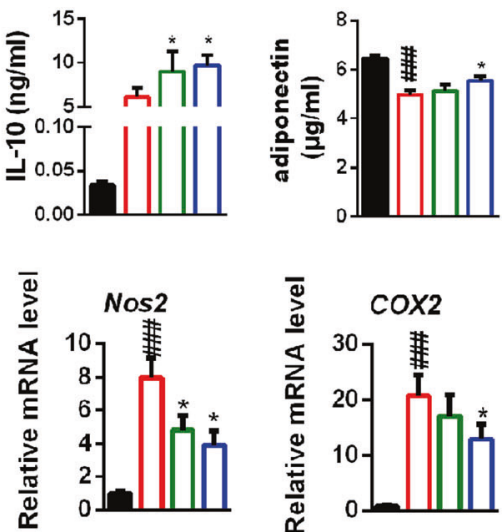

e
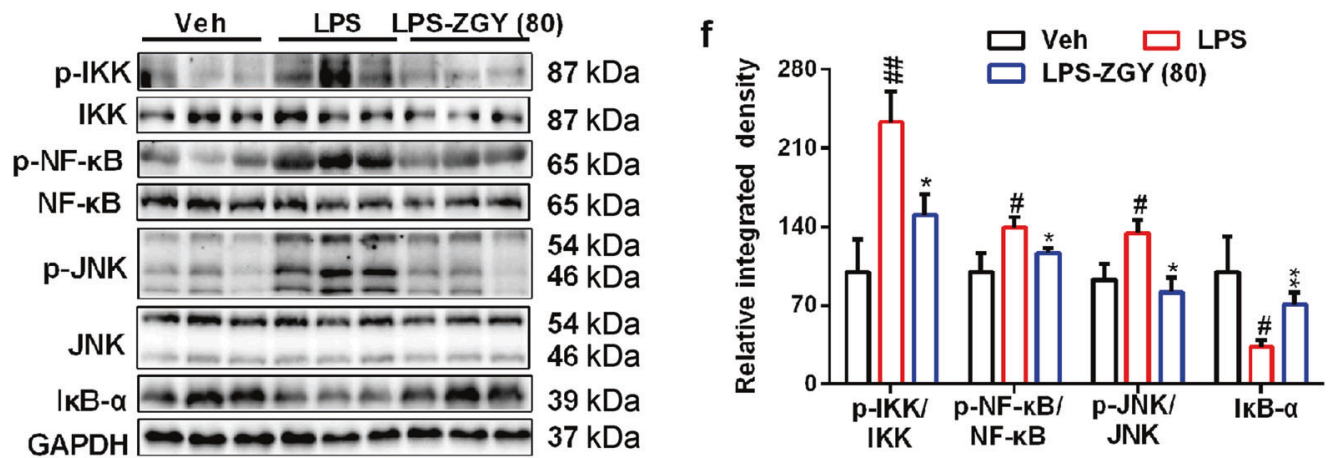

Fig. 2 ZGY inhibits acute adipose inflammatory responses induced by intraperitoneal injection of LPS in C57BL/6J mice. a C57BL/6J mice, weighing $20-22 \mathrm{~g}$ were administered 40 and $80 \mathrm{mg} / \mathrm{kg}$ ZGY via oral gavage for 18 days. At the end of day 18 , the mice were treated by intraperitoneal injection with LPS $(30 \mathrm{mg} / \mathrm{kg})$, and $6 \mathrm{~h}$ after LPS injection, the animals were killed $(n=8-10)$. The concentration of cytokines in the plasma was detected by ELISA. b RT-PCR analysis of M1 marker gene expression in eWAT after treatment with LPS and/or ZGY ( $n=8-10)$. c RT-PCR analysis of M2 marker gene expression in adipose tissue $(n=8-10)$. d Relative expression of adiponectin was quantified by RT-PCR (normalized to GAPDH) $(n=8-10)$. e The protein expression of inflammatory pathways was assessed by Western blotting. $\mathbf{f}$ The protein levels were quantified by densitometry analysis $(n=8-10)$. The data are presented as the means \pm SEM. Two-tailed Student's $t$-test. ${ }^{*} P<0.05$, ${ }^{* *} P<$ 0.01 compared with the LPS group; ${ }^{\#} P<0.05$, ${ }^{\# \#} P<0.01$, ${ }^{\# \#} P<0.001$ compared between the Veh and LPS groups.

ZGY treatment alleviates inflammation state in epididymal adipose tissue of HFD mice

Obese subjects and animal models of insulin resistance are characterized by increased plasma levels of TNF-a, IL-1 $\beta, \mathrm{IL}-6$, and MCP-1 [4, 8, 9]. Next, we investigated the effect of ZGY on systemic and adipose tissue inflammation in HFD mice. Our data showed that oral administration of ZGY in HFD mice elevated the plasma levels of anti-inflammatory cytokines, including adiponectin and IL-10 which are positively correlated with insulin resistance; the plasma levels of IL-1 $\beta, \mathrm{IL}-6$, and MCP-1, which are proinflammatory cytokines and are negatively correlated with insulin resistance, were elevated in HFD mice and reduced by supplementation with ZGY (Fig. 4a). However, there was no change in the level of TNF-a in any of the groups (Fig. 4a).

It is well established that HFD increases the accumulation of macrophages and proinflammatory M1 macrophage polarization in adipose tissue, which are implicated in the development of obesity-related insulin resistance [13]. Therefore, we investigated the effects of ZGY on eWAT inflammation. To identify and quantify macrophages within eWAT, we immunohistochemically stained sections for the F4/80 antigen, which is a marker that is specific for mature macrophages. We calculated the percentage of F4/80expressing cells in HFD mouse eWAT. We found that oral ZGY reduced the percentage of F4/80-positive cells within HFD mice eWAT (Fig. 4b, c).

Next, we determined the mRNA expression levels of macrophage marker genes, M1 proinflammatory genes, and M2 antiinflammatory genes in eWAT by RT-PCR. These data demonstrated that ZGY decreased the expression of the macrophage marker genes $F 4 / 80$ and $C D 68$, reduced the expression of the M1 marker genes TNF- $a, I L-1 \beta$, and $M C P-1$ (Fig. $4 \mathrm{~d}$ ), and increased the mRNA levels of the M2-associated genes $I L-10, Y m 1$, and Fizzl in HFD mouse eWAT (Fig. 4e). To further elucidate the potential mechanism of ZGY, we detected the effect of ZGY on the IKK/ $N F-K B$ and JNK signaling pathways by Western blotting. The enhanced phosphorylation of inflammatory signaling pathways in HFD mouse eWAT was alleviated by ZGY treatment (Fig. 4f, g). Many proinflammatory cytokines upregulate suppressor of cytokine signaling 3 (SOCS3) and protein tyrosine phosphatase 1B (PTP1B), and elevated expression of these proteins in insulin target 
a

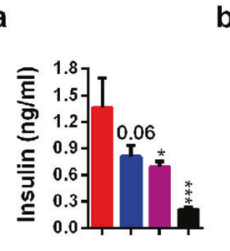

b

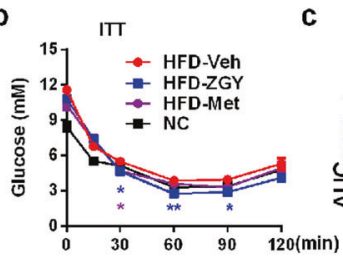

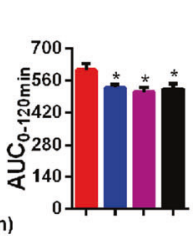

d

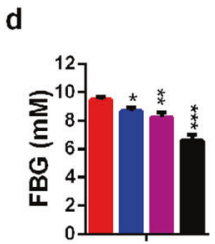

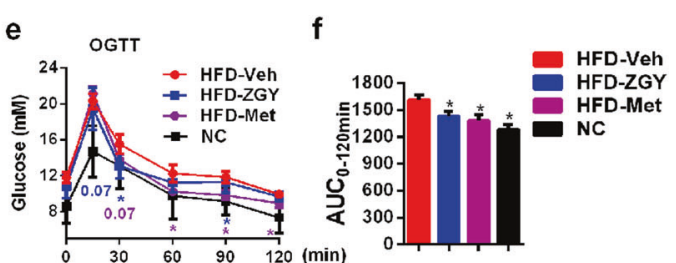

g



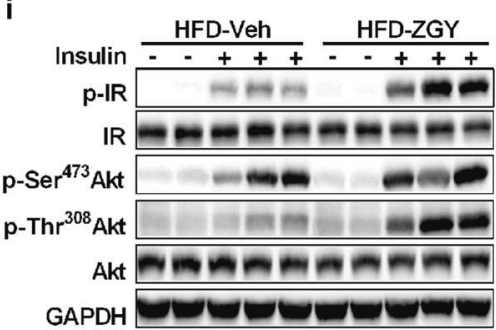

k

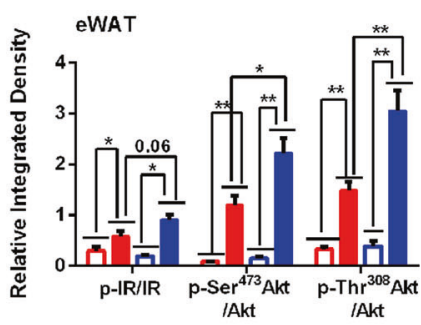

j

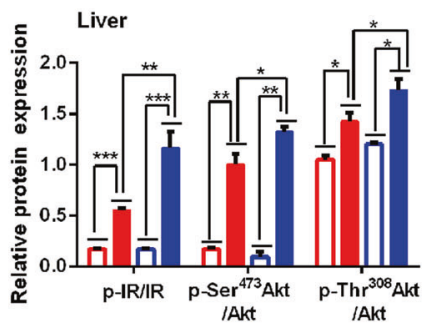

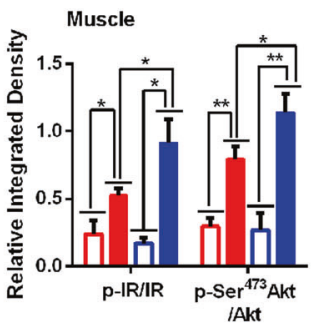

Fig. 3 Chronic ZGY treatment ameliorates insulin resistance in high-fat diet-induced mice. Obese C57BL/6J mice were induced by a highfat diet for 10 weeks and were treated with $80 \mathrm{mg} / \mathrm{kg}$ ZGY (HFD-ZGY) and $250 \mathrm{mg} / \mathrm{kg}$ Met (HFD-Met) for another 6 weeks ( $n=6-8)$. a Fasting plasma insulin levels of mice after 4 week of treatment $(n=6-8)$. b Insulin tolerance test of mice at 4 weeks of treatment ( $n=6-8)$. c The AUC from 0 to $120 \mathrm{~min}$ was calculated $(n=6-8)$. d Fasting blood glucose levels of mice after 5 weeks of treatment. e Glucose tolerance test of mice at 5 weeks of treatment $(n=6-8)$. $\mathbf{f}$ The AUC from 0 to $120 \mathrm{~min}$ was calculated $(n=6-8)$. $\mathbf{g}$, $\mathbf{i}$, $\mathbf{k}$ The expression levels of insulin signal components stimulated by insulin in eWAT $(\mathbf{g})$, the liver (i), and muscle (k) of mice after 6 weeks of treatment were determined by Western blot analysis. h, j, I Relative protein levels were determined by densitometric quantification of the immunoblots shown in ( $\mathbf{g}$, i, $\mathbf{k}$ ). eWAT (h) shown in (g), the liver (j) shown in (i), and muscle (I) shown in (k) $(n=6-8)$. The data are presented as the means \pm SEM. Two-tailed Student's $t$-test. ${ }^{*} P<0.05,{ }^{* *} P<0.01,{ }^{* * *} P<0.001$ compared with the HFD-Veh group.

tissues induces insulin resistance [27-29]. ZGY inhibited the gene expression of PTP1B and SOCS3 and increased the mRNA levels of adiponectin and IRS1 in HFD mouse eWAT (S Fig. 2d). These data confirm that ZGY treatment ameliorates local inflammation in adipose tissue, which may contribute to improved insulin sensitivity.

ZGY alleviates TNF-a-induced inflammatory responses and improves adipocyte sensitivity to insulin

Our studies demonstrated that oral administration of ZGY improved the inflammatory state and insulin resistance in adipose tissue of HFD mice. We wondered whether ZGY acts directly on adipocytes. We stimulated mature 3T3-L1 adipocytes with TNF-a alone or in combination with ZGY and investigated the expression of proinflammatory genes and activation of inflammatory pathways and insulin signaling transduction pathways [30, 31]. The results showed that ZGY repressed TNF-a-induced expression of proinflammatory genes, such as $I L-1 \beta, I L-6$, and $M C P-1$ (Fig. 5a). ZGY decreased TNF-a-induced phosphorylation of IKK, NF-KB, and JNK, and protected the protein level of IKB- $a$ in differentiated 3T3L1 cells (Fig. 5b, c). Insulin-induced phosphorylation of IR and Akt was reduced by incubation with TNF- $a$, and ZGY treatment enhanced the phosphorylation of IR, Ser ${ }^{473} \mathrm{Akt}$, and $\mathrm{Thr}^{308} \mathrm{Akt}$ in mature 3T3-L1 cells, which indicated that ZGY treatment increased intracellular signaling responses to insulin (Fig. 5d, e).

Furthermore, in isolated and differentiated inguinal white adipose tissue stromal vascular fraction (iWAT-SVF) cells from mice, we also observed that ZGY inhibited TNF-a-induced expression of the proinflammatory genes TNF- $a, I L-6$, and $M C P-1$ in mature iWAT-SVF cells (Fig. 5f). ZGY also inactivated the IKK/NF$K B$ and JNK signaling pathways (S Fig. 3a) and increased the phosphorylation of IR, Ser ${ }^{473} \mathrm{Akt}$, and $\mathrm{Thr}^{308} \mathrm{Akt}$ in TNF-a-treated mature iWAT-SVF cells (S Fig. 3b). Collectively, these data show that ZGY ameliorates TNF-a-induced adipocyte inflammatory responses and contributes to protecting insulin sensitivity in differentiated adipocytes via the TNF-a-dependent pathway.

ZGY suppresses 3T3-L1 adipocyte inflammation and insulin resistance induced by conditioned medium (CM) from LPS-activated RAW 264.7 macrophages

Emerging lines of evidence have shown that secreted factors from macrophages induce inflammatory responses and insulin resistance in adipocytes [30, 32, 33]. Blocking the crosstalk between adipocytes and macrophages could be a promising strategy for the treatment of adipose tissue inflammation and insulin resistance $[31,34,35]$. Studies have indicated that ZGY inhibits LPS-induced macrophage inflammatory responses [16]. Our data also showed that ZGY inhibited LPS-induced inflammatory gene expression and inactivated the inflammatory signaling pathway in RAW 264.7 macrophages (S Fig. 4). To ascertain the effects of ZGY on the interaction between adipocytes and macrophages, we used an indirect coculture system to elucidate whether ZGYmediated suppression of proinflammatory gene expression and secretion in macrophages could rescue insulin sensitivity in 
a

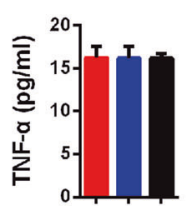

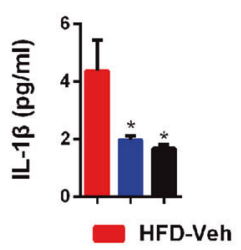


b

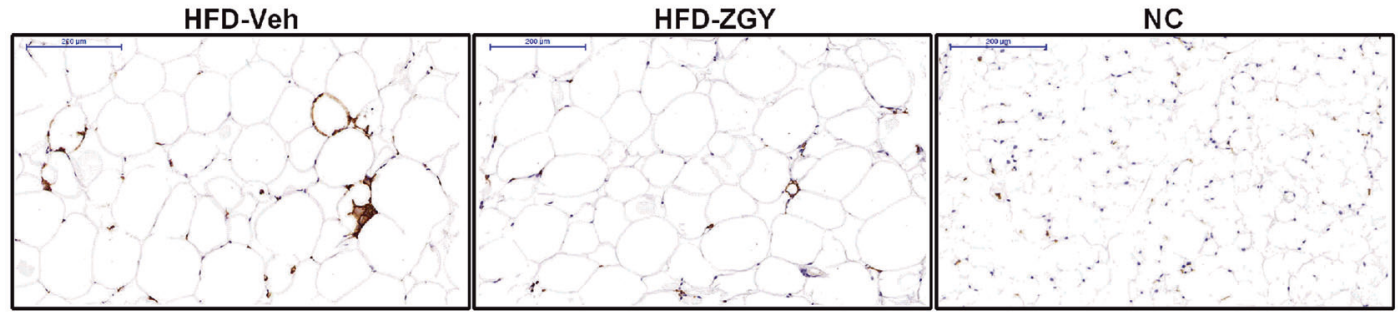

C

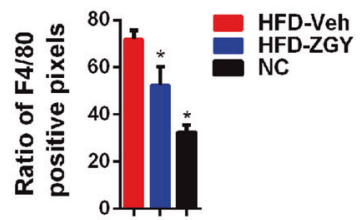

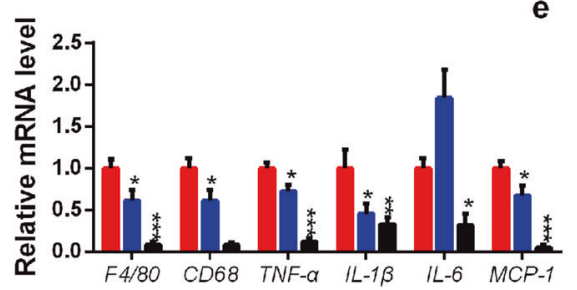



f

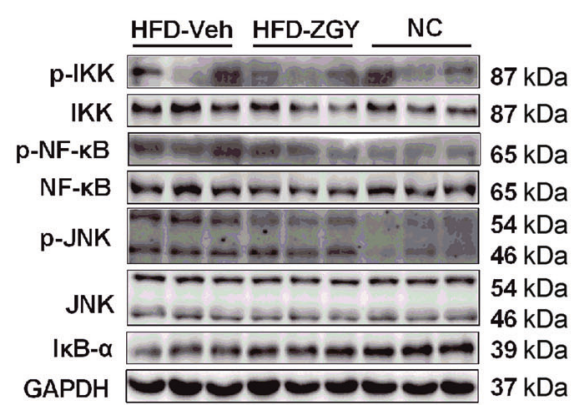

g

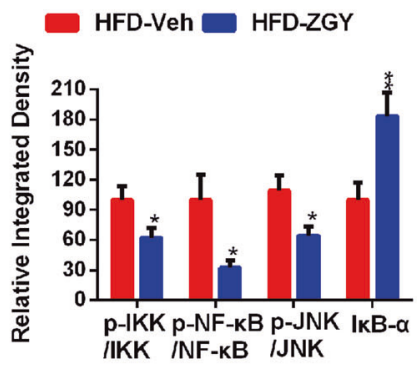

Fig. 4 ZGY treatment alleviates inflammation in epididymal adipose tissue of HFD mice. a Plasma cytokines were measured using ELISA $(n=6-8)$. b F4/80 immunohistochemical analysis of eWAT; scale bar $=200 \mu \mathrm{m}(n=6-8)$. c The ratio of all F4/80-positive pixels were counted in each fraction of eWAT shown in (b) $(n=6-8)$. $\mathbf{d}$ Expression of macrophage and M1 proinflammatory genes in eWAT was quantified by RT-PCR $(n=6-8)$. e The relative mRNA levels of M2 anti-inflammatory genes in eWAT of mice (normalized to GAPDH) $(n=6-8)$. $\mathbf{f}$ Western blot analysis of inflammatory signaling protein expression in eWAT of HFD mice. g Relative protein expression levels were determined by densitometric quantification of the immunoblots shown in $(\mathbf{f})(n=6-8)$. The data are presented as the means \pm SEM. Two-tailed Student's $t$-test. ${ }^{*} P<0.05$, ${ }^{* *} P<0.01,{ }^{* *} P<0.001$ compared with the HFD-Veh group.

adipocytes. We incubated mature 3T3-L1 adipocytes with conditioned medium (CM) derived from LPS-activated RAW 264.7 macrophages in the presence or absence of ZGY. The addition of CM significantly increased the expression of proinflammatory cytokines such as TNF- $a, I L-1 \beta, I L-6$, and MCP-1 and enhanced the levels of secreted TNF-a, IL-6, and MCP-1, as previously observed $[30,33,35]$. As expected, pretreatment of macrophages with ZGY downregulated the mRNA levels of TNF- $a, I L-1 \beta, I L-6$, and MCP-1 (Fig. 6a) and suppressed the release of TNF-a, IL-6, and MCP-1 (Fig. 6b). The mRNA and secreted protein levels of adiponectin were depleted when 3T3-L1 adipocytes were incubated with CM from macrophages that were stimulated with LPS-alone, which was consistent with a previous study [36]. In our study, we found that the decrease in adiponectin mRNA levels was reversed by ZGY-treated CM (Fig. 6a), and the secretion of adiponectin showed an increasing trend, although there was no significant difference (Fig. 6b).

Moreover, we also proved that ZGY inhibited the phosphorylation of IKK, NF-KB, and JNK and increased the protein level of IKB$a$, possibly by inhibiting the degradation of $/ \kappa B-a$ that was induced by CM containing LPS (S Fig. 5a). In addition, we also found that ZGY increased the phosphorylation of IR, Ser ${ }^{473} \mathrm{Akt}$, and $\mathrm{Thr}^{308} \mathrm{Akt}$, which was attenuated by CM containing LPS (S Fig. 5b). These findings suggest that ZGY alters the repertoire of macrophage cytokines, leading to improvements in adipocyte inflammatory responses and resistance to insulin.

\section{DISCUSSION}

Insulin resistance is a central mechanism in obesity-associated diseases, such as type 2 diabetes [2]. Obesity is characterized by chronic low-grade inflammation and is strongly linked to insulin resistance [1]. Adipose tissue is the main source and initiation site of inflammatory responses [37]. In the obese state, infiltrated macrophages interact with adipocytes, which promotes macrophage M1 polarization, establishes a vicious cycle that exacerbates inflammatory responses and contributes to local and systemic insulin resistance in an endocrine and paracrine fashion [5, 9]. It has been reported that omega- 3 fatty acids reduce adipose tissue macrophages in human subjects with insulin resistance [38]. 
a
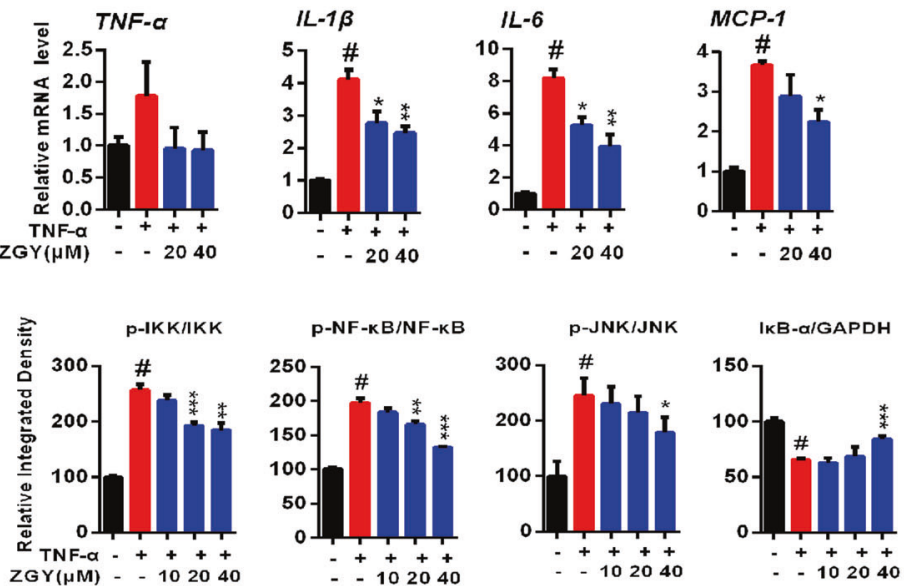

d

Insulin - +-+++++

$\begin{array}{ccccccccc}\text { ZGF }(\mu \mathrm{M}) & - & - & + & + & + & + & + & + \\ & - & - & - & - & 2.5 & 10 & 20 & 40\end{array}$

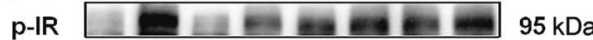

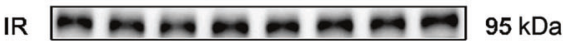

p-Ser ${ }^{473}$ Akt $\square 60 \mathrm{kDa}$

p-Thr ${ }^{308}$ Akt $\square-\infty 0 m 60$

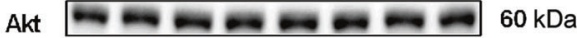

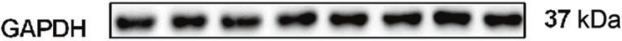

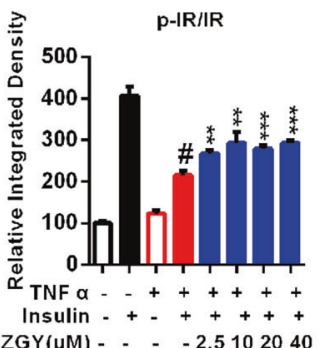

b

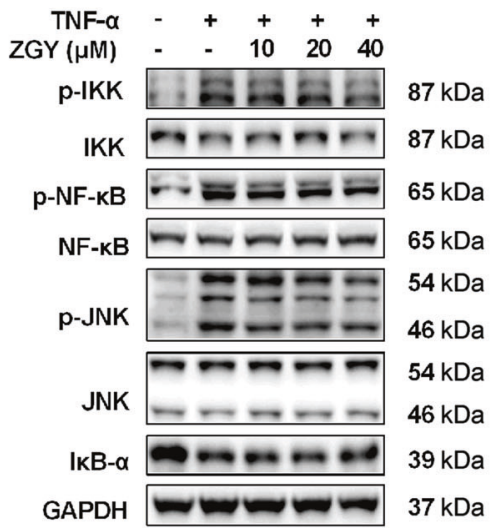

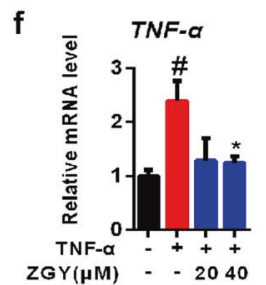
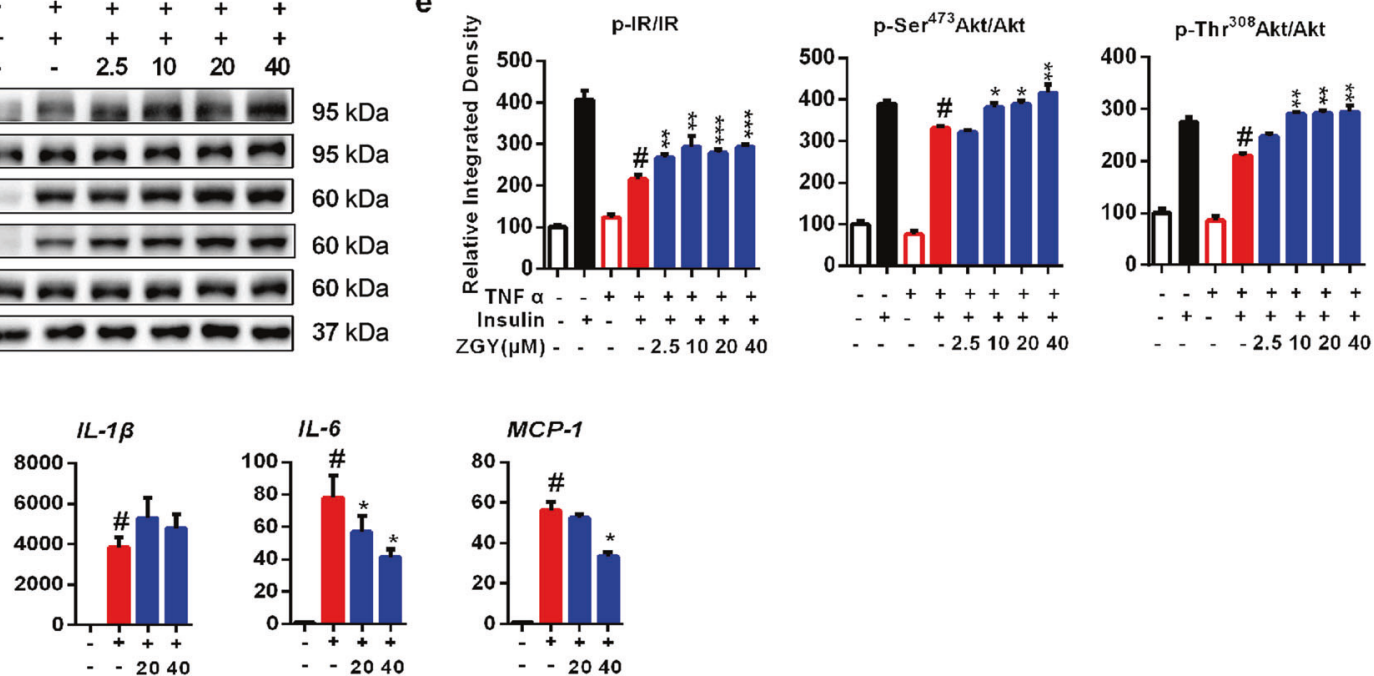

Fig. 5 ZGY alleviates TNF-a-induced inflammatory responses and improves adipocyte sensitivity to insulin. a Differentiated 3T3-L1 cells were treated with TNF- $\alpha(100 \mathrm{ng} / \mathrm{mL})$ in the absence or presence of ZGY at the indicated concentrations for $24 \mathrm{~h}$. The relative mRNA level of inflammatory genes was quantified by RT-PCR (normalized to 36b4) $(n=4)$. b Mature 3T3-L1 cells were treated with ZGY for $24 \mathrm{~h}$ before TNF- $\alpha$ $(100 \mathrm{ng} / \mathrm{mL})$ stimulation for $30 \mathrm{~min}$. Protein expression of inflammatory pathways was analyzed by Western blotting. c Relative protein expression levels were determined by densitometric quantification of the immunoblots shown in $(\mathbf{b})(n=3)$. d Mature 3T3-L1 cells were treated with TNF- $\alpha(100 \mathrm{ng} / \mathrm{mL})$ alone or with ZGY for $24 \mathrm{~h}$, followed by insulin $(100 \mathrm{nM})$ stimulation for 30 min. The protein levels of insulin signaling components were determined by Western blot analysis. e Relative protein expression levels were determined by densitometric quantification of the immunoblots shown in (d) $(n=3)$. ${ }^{\#} P<0.05$ compared between the second and fourth columns; ${ }^{*} P<0.05$, ${ }^{* *} P<0.01$, ${ }_{* * *}^{*}<0.001$ compared with the fourth column. $\mathbf{f}$ iWAT-SVF cells were induced to differentiatiate into white-like adipocytes and were treated with ZGY and/or TNF- $\alpha(100 \mathrm{ng} / \mathrm{mL}$ ) on day 8 . The relative mRNA levels of inflammatory genes were quantified by RT-PCR (normalized to $36 b 4$ ) $(n=4)$. The data are presented as the means \pm SEM. Two-tailed Student's $t$-test. ${ }^{*} P<0.05,{ }^{* *} P<0.01,{ }^{* * *} P<0.001$ compared with the TNF- $\alpha$ group; ${ }^{\#} P<0.05$ compared between negative control and TNF- $\alpha$ group.

Thiazolidinediones improve diabetic human insulin sensitivity by reducing adipose tissue macrophage levels [39]. Glucagon-like peptide- 1 inhibits adipose tissue macrophage infiltration and inflammation in an obese mouse model of diabetes [40]. Glucagon-like peptide- 1 analog therapy directly modulates innate immune-mediated inflammation in individuals with type 2 diabetes mellitus [41].

The rhizome of Anemarrhena asphodeloides is a typical herbal medicine that improves learning and memory in rats and inhibits inflammation. Its constituent, mangiferin, inhibits the NF-KB signaling pathway and ameliorates inflammatory diseases such as colitis. Furostanol timosaponin B inhibits NF-KB activation in macrophages. Timosaponin All and its metabolite sarsasapogenin (ZGY) ameliorate colitis in mice by inhibiting NF-KB and MAPK activation and restoring the Th17/Treg cell balance, and experiments have shown that the anti-inflammatory effects of ZGY are more potent than those of timosaponin Alll [16]. A novel synthetic derivative of ZGY inhibits LPS-induced inflammatory responses in macrophages in vitro and alleviates dimethylbenzene-induced ear edema in mice [42]. ZGY markedly ameliorates diabetic nephropathy in rats via inhibition of NLRP3 inflammasome activation [43].

Recently, we found that ZGY preferentially accumulates in adipose tissue after a single oral administration. Notably, the effects of ZGY on obesity-induced adipose tissue inflammation and insulin resistance have not been investigated.

LPS is a strong stimulator of the release of several cytokines, and HFD feeding augments the concentration of plasma LPS by changing the gut microbiota and is sufficient to induce inflammation and insulin resistance $[26,44,45]$. In our study, we found that pretreatment of C57BL/6J mice with ZGY suppressed LPS-induced inflammatory responses in vivo, as evidenced by the reduced levels of inflammatory cytokines and increased levels of anti-inflammatory cytokines. These findings further verified the anti-inflammatory effect of ZGY. 

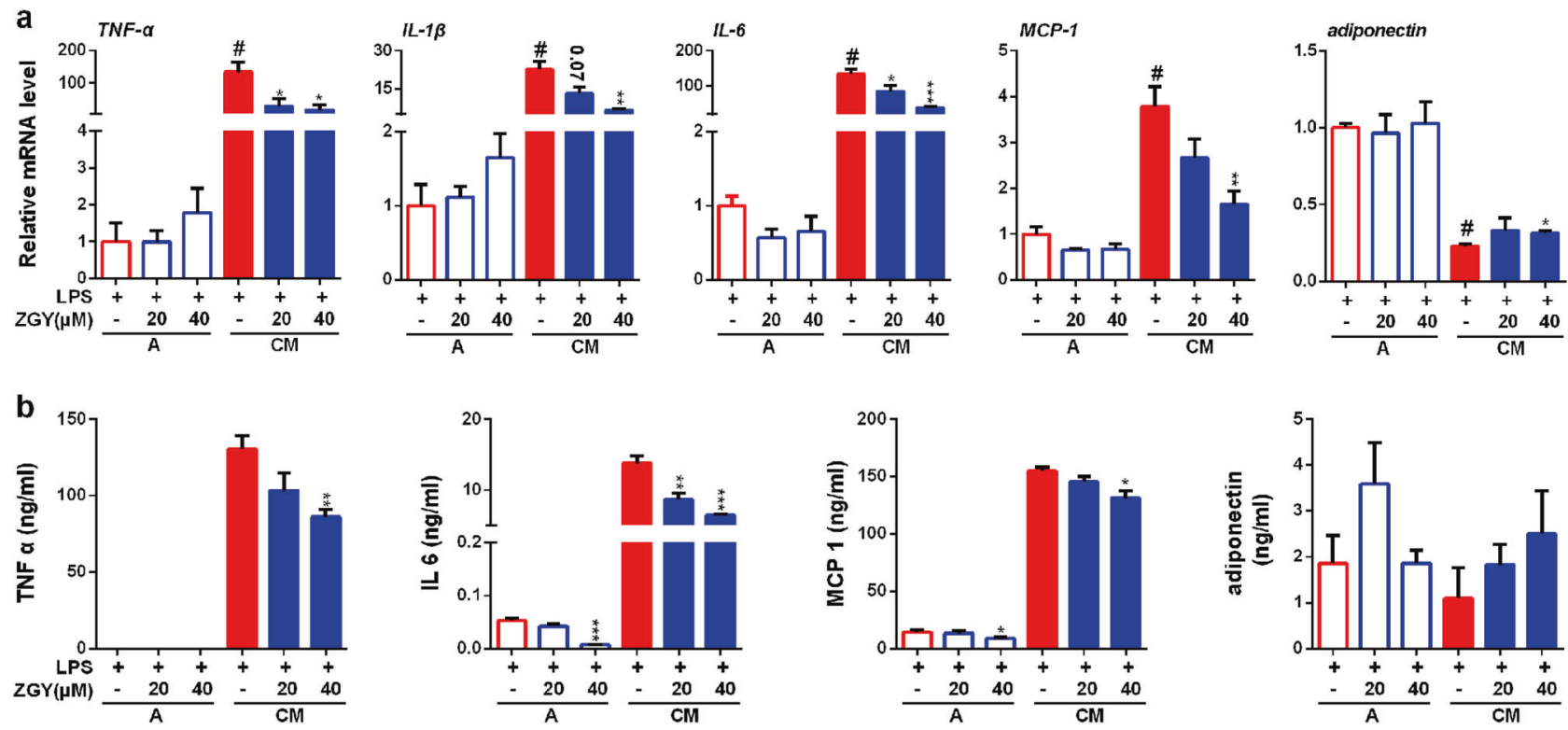

Fig. 6 ZGY suppresses 3T3-L1 adipocyte inflammation and insulin resistance induced by conditioned medium from LPS-activated RAW 264.7 macrophages. Differentiated 3T3-L1 adipocytes were incubated for $24 \mathrm{~h}$ with CM from RAW 264.7 macrophages that were treated with LPS $(100 \mathrm{ng} / \mathrm{mL})$ without or with ZGY. Cultured medium containing the same concentration of LPS and/or ZGY-treated 3T3-L1 cells were used as a negative control and were named $\mathrm{A}$. a Relative mRNA levels of M1 proinflammatory genes and adiponectin in each group, as measured by RT-PCR (normalized to 36b4). b The cytokine secretion in the coculture medium was measured by ELISA $(n=3-4)$. The data are presented as the means \pm SEM. Two-tailed Student's $t$-test. ${ }^{*} P<0.05,{ }^{* *} P<0.01,{ }^{* *} P<0.001$ compared with the first or fourth row; ${ }^{\#} P<0.05$ compared between the first and fourth rows.

Adipose tissue is a prominent source of various cytokines [37]. The IKK/NF-KB and JNK signaling pathways promote inflammatory cytokine gene expression and secretion and have central roles in promoting tissue inflammation [1]. Many studies have shown that activation of IKK/NF-KB and JNK signaling blocks insulin action by desensitizing insulin signaling. Moreover, pharmacological and genetic downregulation of inflammatory pathway activation in mice confers protection against HFDinduced insulin resistance [14]. In our study, we found that ZGY inhibited the expression of inflammatory cytokines and activation of the IKK/NF-KB and JNK signaling pathways in HFD mouse epididymal adipose tissue. These results are similar to those of Lim et al., who found that oral ZGY changed the expression of inflammatory cytokines in mouse colitis and the secretion in mice that were stimulated with TNBS [16]. These findings indicate that ZGY acts on adipose tissues.

Next, we used an HFD-induced obesity mouse model to assess the effect of ZGY on insulin resistance. In our study, we proved for the first time that ZGY ameliorated HFD-induced insulin resistance in vivo. This result was associated with improvements in systemic and epididymal adipose tissue inflammation. Expansion of adipose tissue in obesity leads to increased macrophage infiltration and dysfunctional secretion in adipose tissue [2]. In addition, obese adipose tissue macrophages undergo a phenotypic switch from an anti-inflammatory M2 polarization state to a proinflammatory $M 1$ polarization state $[46,47]$. Lee et al. proved that inhibiting adipose tissue macrophage infiltration can improve insulin resistance in obese mice [39]. Lumeng et al. and Saltiel et al. showed that alternatively activated M2 macrophages contribute to maintaining insulin sensitivity via the expression and secretion of anti-inflammatory cytokines, and classically activated M1 macrophages induce insulin resistance via the expression and secretion of proinflammatory cytokines [13, 48]. Consistent with their results, our data showed that macrophages were reduced in ZGY-treated HFD mouse eWAT as analyzed by immunohistochemical staining and RT-PCR. Moreover, ZGY inhibited the expression of M1 macrophage marker genes and increased the mRNA level of M2 macrophage marker genes in eWAT.

In vitro, we also confirmed that ZGY inhibited LPS-induced macrophage inflammatory responses, which was consistent with reports by Lim et al. [16]. To determine whether there were direct effects of ZGY on adipocytes, we examined the effects of ZGY on inflammatory responses and insulin resistance of TNF-a-induced mature 3T3-L1 and iWAT-SVF cells. Our data showed that ZGY inhibited inflammatory gene expression and activation of IKK/NF$\mathrm{KB}$ and JNK signaling. Furthermore, ZGY enhanced insulin signaling pathway sensitivity in TNF-a-treated adipocytes. These results were similar to those of adipose tissue and revealed that ZGY directly acts on adipose tissue and adipocytes. ZGY improved adipose tissue and adipocyte insulin resistance possibly by alleviating inflammatory responses.

Several lines of evidence have clearly shown that the interactions between macrophages and adipocytes induce a vicious cycle, thereby exacerbating adipose tissue inflammation and reducing insulin sensitivity $[30,49]$. Thus, targeting interactions between adipocytes and macrophages to mitigate chronic inflammation in obesity may be a therapeutic strategy for insulin resistance [50]. Ceppo et al. proved that pharmacological inhibition of Tpl2 improved insulin resistance induced by coculturing adipocytes and macrophages by inhibiting inflammatory gene expression and secretion [33]. Iwashita et al. showed that the angiotensin II receptor blocker valsartan reversed adipocyte and macrophage coculture-induced insulin resistance by inactivating NF-KB and JNK proteins and inhibiting inflammatory gene expression and secretion [31]. Mazur-Bialy et al. suggest that riboflavin supplementation induces functional changes in adipocyte-macrophage cocultures and leads to a reduction in the intensity of their proinflammatory, insulin resistance and diabetic activities by suppressing NF-KB transcriptional activity [34].

We wondered whether ZGY halts the vicious cycle involving macrophages and adipocytes. We used indirect coculture models (adipocytes treated with conditioned medium from LPS-activated macrophages) to mimic the interaction of adipocytes and 
macrophages in obese adipose tissue. In the coculture systems, the mRNA expression and secretion of proinflammatory adipokines were increased compared to those of the negative control. These observations are consistent with previous studies $[33,35,49]$, and these changes were restored by ZGY treatment. In addition, the activation of inflammatory signaling pathways and attenuation of the insulin signaling pathway in the coculture system, which were similar to the results of Suganami et al. $[49,51]$, were also changed by ZGY.

However, in our work, some questions remain unclear. Although we and other researchers have proven that $Z G Y$ inhibits inflammatory responses through inactivation of the IKK/NF-KB and JNK signaling pathways, the direct target of ZGY should be further explored. In addition, we did not measure the levels of M1 and M2 macrophages precisely, only measuring the inflammatory gene expression in adipocytes and in white adipose tissue from HFD mice, and the change in cytokine secretion in vivo and in vitro suggested that there may be reprogramming of macrophages in vivo and in vitro. Thus, whether ZGY regulates and mediates the polarization of macrophages is another question worth studying.

\section{CONCLUSION}

In summary, our results demonstrate for the first time that the oral agent ZGY alleviates insulin resistance in HFD-induced obese mice and ameliorates adipose tissue inflammation. The possible mechanism may be through perturbing the deleterious crosstalk between adipocytes and macrophages in adipose tissue in obesity by inactivating the IKK/NF-KB and JNK signaling pathways. Importantly, our studies indicate that ZGY may be a potential treatment for chronic inflammation-related metabolic diseases. Moreover, ZGY is the gut metabolite of timosaponin Alll and has a more potent effect on TNBS-induced colitis in mice [16]. In our study, although we did not examine the effect of timosaponin Alll on HFD-induced insulin resistance, our results and other data indicate that timosaponin Alll, the rhizome of Anemarrhena asphodeloides, and its constituents, mangiferin and furostanol timosaponin B, may improve insulin resistance in HFD-induced mice by ameliorating the inflammatory response and have preventative and therapeutic effects on chronic inflammatoryrelated metabolic diseases.

\section{ACKNOWLEDGEMENTS}

This work was supported by grants from the Ministry of Science and Technology (2018ZX09711002-018 and 2016YFC1305505) and the National Natural Science Foundation of China (81673493).

\section{AUTHOR CONTRIBUTIONS}

YYY contributed to the hypothesis, study design, data analysis, experiments, and preparation of the paper; SCC, TNZ, HJM, ZFX, HWJ, YFL, and KXZ performed the experiments, analyzed the data, provided conceptual advice and technical expertise, and edited the paper; HCG provided all the ZGY samples for the in vitro and in vivo experiments. $\mathrm{JL}$ and JYL conceived and supervised the study; and all authors reviewed and approved the final version of the paper.

\section{ADDITIONAL INFORMATION}

The online version of this article (https://doi.org/10.1038/s41401-020-0427-1) contains supplementary material, which is available to authorized users.

Competing interests: The authors declare no competing interests.

\section{REFERENCES}

1. Johnson AM, Olefsky JM. The origins and drivers of insulin resistance. Cell. 2013;152:673-84.
2. Jung UJ, Choi MS. Obesity and its metabolic complications: the role of adipokines and the relationship between obesity, inflammation, insulin resistance, dyslipidemia and nonalcoholic fatty liver disease. Int J Mol Sci. 2014;15:6184-223.

3. Lackey DE, Olefsky JM. Regulation of metabolism by the innate immune system. Nat Rev Endocrinol. 2016;12:15-28.

4. Lumeng CN, Saltiel AR. Inflammatory links between obesity and metabolic disease. J Clin Invest. 2011;121:2111-7.

5. Shoelson SE, Herrero L, Naaz A. Obesity, inflammation, and insulin resistance. Gastroenterology. 2007;132:2169-80.

6. Reinehr T, Roth CL. Inflammation markers in type 2 diabetes and the metabolic syndrome in the pediatric population. Curr Diab Rep. 2018;18:131.

7. Hotamisligil GS. Inflammation and metabolic disorders. Nature. 2006;444:860-7.

8. Gregor MF, Hotamisligil GS. Inflammatory mechanisms in obesity. Annu Rev Immunol. 2011;29:415-45.

9. Sun S, Ji Y, Kersten S, Qi L. Mechanisms of inflammatory responses in obese adipose tissue. Annu Rev Nutr. 2012;32:261-86.

10. Olefsky JM, Glass CK. Macrophages, inflammation, and insulin resistance. Annu Rev Physiol. 2010;72:219-46.

11. Hotamisligil GS. Foundations of immunometabolism and implications for metabolic health and disease. Immunity. 2017;47:406-20.

12. de Luca C, Olefsky JM. Inflammation and insulin resistance. FEBS Lett. 2008;582:97-105.

13. Lumeng CN, Bodzin JL, Saltiel AR. Obesity induces a phenotypic switch in adipose tissue macrophage polarization. J Clin Invest. 2007;117:175-84.

14. Donath MY, Shoelson SE. Type 2 diabetes as an inflammatory disease. Nat Rev Immunol. 2011;11:98-107.

15. Kite GC, Porter EA, Simmonds MS. Chromatographic behaviour of steroidal saponins studied by high-performance liquid chromatography-mass spectrometry. J Chromatogr A. 2007;1148:177-83.

16. Lim SM, Jeong JJ, Kang GD, Kim KA, Choi HS, Kim DH. Timosaponin All and its metabolite sarsasapogenin ameliorate colitis in mice by inhibiting NF-kappaB and MAPK activation and restoring Th17/Treg cell balance. Int Immunopharmacol. 2015;25:493-503.

17. Huang XF, Lin YY, Kong LY. Steroids from the roots of Asparagus officinalis and their cytotoxic activity. J Integr Plant Biol. 2008;50:717-22.

18. Hu H, Zhang R, Zhang Y, Xia Z, Hu Y. Role of CREB in the regulatory action of sarsasapogenin on muscarinic $M_{1}$ receptor density during cell aging. FEBS Lett. 2010;584:1549-52

19. Sy L-K, Lok C-N, Wang J-Y, Liu Y, Cheng L, Wan P-K, et al. Identification of "sarsasapogenin-aglyconed" timosaponins as novel A $\beta$-lowering modulators of amyloid precursor protein processing. Chem Sci. 2016;7:3206-14.

20. Wu L, Zhang L, Li B, Jiang H, Duan Y, Xie Z, et al. AMP-activated protein kinase (AMPK) regulates energy metabolism through modulating thermogenesis in adipose tissue. Front Physiol. 2018;9:122.

21. Fu L, Zhu P, Qi S, Li C, Zhao K. MicroRNA-92a antagonism attenuates lipopolysaccharide (LPS)-induced pulmonary inflammation and injury in mice through suppressing the PTEN/AKT/NF-kappaB signaling pathway. Biomed Pharmacother. 2018;107:703-11.

22. Belegri E, Eggels L, la Fleur SE, Boelen A. One-week exposure to a free-choice high-fat high-sugar diet does not interfere with the lipopolysaccharide-induced acute phase response in the hypothalamus of male rats. Front Endocrinol. 2018;9:186.

23. Freitas Lima LC, Braga VA, do Socorro de Franca Silva M, Cruz JC, Sousa Santos $\mathrm{SH}$, de Oliveira Monteiro MM, et al. Adipokines, diabetes and atherosclerosis: an inflammatory association. Front Physiol. 2015;6:304.

24. Galic S, Oakhill JS, Steinberg GR. Adipose tissue as an endocrine organ. Mol Cell Endocrinol. 2010;316:129-39.

25. Guilherme A, Virbasius JV, Puri V, Czech MP. Adipocyte dysfunctions linking obesity to insulin resistance and type 2 diabetes. Nat Rev Mol Cell Biol. 2008;9:367-77.

26. Cani PD, Amar J, Iglesias MA, Poggi M, Knauf C, Bastelica D, et al. Metabolic endotoxemia initiates obesity and insulin resistance. Diabetes. 2007;56:1761-72.

27. Tanti JF, Ceppo F, Jager J, Berthou F. Implication of inflammatory signaling pathways in obesity-induced insulin resistance. Front Endocrinol. 2012;3:181.

28. Ormazabal P, Scazzocchio B, Vari R, Santangelo C, D'Archivio M, Silecchia G, et al. Effect of protocatechuic acid on insulin responsiveness and inflammation in visceral adipose tissue from obese individuals: possible role for PTP1B. Int J Obes. 2018;42:2012-21.

29. Elchebly M, Payette P, Michaliszyn E, Cromlish W, Collins S, Loy AL, et al. Increased insulin sensitivity and obesity resistance in mice lacking the protein tyrosine phosphatase-1B gene. Science. 1999;283:1544-8.

30. Lumeng CN, Deyoung SM, Saltiel AR. Macrophages block insulin action in adipocytes by altering expression of signaling and glucose transport proteins. Am J Physiol Endocrinol Metab. 2007;292:E166-74.

31. Iwashita M, Sakoda H, Kushiyama A, Fujishiro M, Ohno H, Nakatsu Y, et al. Valsartan, independently of $\mathrm{AT}_{1}$ receptor or PPARgamma, suppresses LPS-induced 
macrophage activation and improves insulin resistance in cocultured adipocytes. Am J Physiol Endocrinol Metab. 2012;302:E286-96.

32. Yamashita A, Soga $Y$, Iwamoto $Y$, Yoshizawa S, Iwata $H$, Kokeguchi S, et al. Macrophage-adipocyte interaction: marked interleukin-6 production by lipopolysaccharide. Obesity. 2007;15:2549-52.

33. Ceppo F, Berthou F, Jager J, Dumas K, Cormont M, Tanti JF. Implication of the Tpl2 kinase in inflammatory changes and insulin resistance induced by the interaction between adipocytes and macrophages. Endocrinology. 2014;155:951-64.

34. Mazur-Bialy Al, Pochec E. Riboflavin reduces pro-inflammatory activation of adipocyte-macrophage co-culture. Potential application of vitamin $B_{2}$ enrichment for attenuation of insulin resistance and metabolic syndrome development. Molecules. 2016;21:1724.

35. Jung YJ, Kim BO, Kwak JH, Pyo S. Inhibitory effect of methyl 2-(4'-methoxy-4'oxobutanamide) benzoate from jerusalem artichoke (Helianthus tuberosus) on the inflammatory paracrine loop between macrophages and adipocytes. J Agric Food Chem. 2016;64:9317-25.

36. Feng $B$, Jiao $P$, Nie $Y$, Kim $T$, Jun $D$, van Rooijen $N$, et al. Clodronate liposomes improve metabolic profile and reduce visceral adipose macrophage content in diet-induced obese mice. PLoS ONE. 2011;6:e24358.

37. Makki K, Froguel P, Wolowczuk I. Adipose tissue in obesity-related inflammation and insulin resistance: cells, cytokines, and chemokines. ISRN Inflamm. 2013;2013:139239.

38. Spencer M, Finlin BS, Unal R, Zhu B, Morris AJ, Shipp LR, et al. Omega-3 fatty acids reduce adipose tissue macrophages in human subjects with insulin resistance. Diabetes. 2013;62:1709-17.

39. Koppaka S, Kehlenbrink S, Carey M, Li W, Sanchez E, Lee DE, et al. Reduced adipose tissue macrophage content is associated with improved insulin sensitivity in thiazolidinedione-treated diabetic humans. Diabetes. 2013;62:1843-54.

40. Lee YS, Park MS, Choung JS, Kim SS, Oh HH, Choi CS, et al. Glucagon-like peptide1 inhibits adipose tissue macrophage infiltration and inflammation in an obese mouse model of diabetes. Diabetologia. 2012;55:2456-68.

41. Hogan $A E$, Gaoatswe G, Lynch L, Corrigan MA, Woods C, O'Connell J, et al Glucagon-like peptide 1 analogue therapy directly modulates innate immune- mediated inflammation in individuals with type 2 diabetes mellitus. Diabetologia. 2013;57:781-84.

42. Dong D, Zhou NN, Liu RX, Xiong JW, Pan H, Sun SQ, et al. Sarsasapogenin-AA13 inhibits LPS-induced inflammatory responses in macrophage cells in vitro and relieves dimethylbenzene-induced ear edema in mice. Acta Pharmacol Sin. 2017;38:699-709.

43. Liu YW, Hao YC, Chen YJ, Yin SY, Zhang MY, Kong L, et al. Protective effects of sarsasapogenin against early stage of diabetic nephropathy in rats. Phytother Res. 2018;32:1574-82.

44. Saad MJ, Santos A, Prada PO. Linking gut microbiota and inflammation to obesity and insulin resistance. Physiology. 2016;31:283-93.

45. Cani PD, Osto M, Geurts L, Everard A. Involvement of gut microbiota in the development of low-grade inflammation and type 2 diabetes associated with obesity. Gut Microbes. 2012;3:279-88.

46. Morris DL, Singer K, Lumeng CN. Adipose tissue macrophages: phenotypic plasticity and diversity in lean and obese states. Curr Opin Clin Nutr Metab Care. 2011;14:341-6.

47. Peterson KR, Cottam MA, Kennedy AJ, Hasty AH. Macrophage-targeted therapeutics for metabolic disease. Trends Pharmacol Sci. 2018;39:536-46.

48. Saltiel AR, Olefsky JM. Inflammatory mechanisms linking obesity and metabolic disease. J Clin Invest. 2017;127:1-4.

49. Suganami T, Nishida J, Ogawa Y. A paracrine loop between adipocytes and macrophages aggravates inflammatory changes: role of free fatty acids and tumor necrosis factor alpha. Arterioscler Thromb Vasc Biol. 2005;25 2062-8.

50. Namkoong S, Sung J, Yang J, Choi Y, Jeong HS, Lee J. Nobiletin attenuates the inflammatory response through heme oxygenase- 1 induction in the crosstalk between adipocytes and macrophages. J Med Food. 2017;20: 873-81.

51. Suganami T, Tanimoto-Koyama K, Nishida J, Itoh M, Yuan X, Mizuarai S, et al. Role of the Toll-like receptor 4/NF-kappaB pathway in saturated fatty acid-induced inflammatory changes in the interaction between adipocytes and macrophages. Arterioscler Thromb Vasc Biol. 2007;27:84-91. 